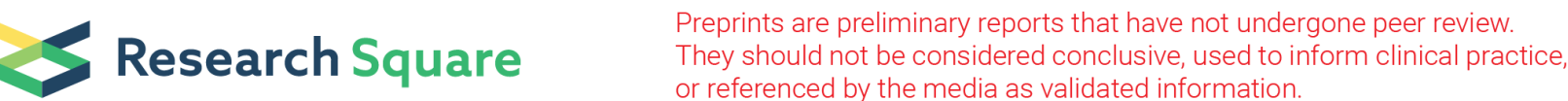

\section{Emergency high ligation in a suspected COVID-19 pediatric patient with incarcerated inguinal hernia: a case report}

\section{Munawir Makkadafi}

Universitas Gadjah Mada Fakultas Kedokteran

\section{Aditya Rifqi Fauzi}

Universitas Gadjah Mada Fakultas Kedokteran

Amsyar Praja

Undata District Hospital, Palu

Kemala Athollah

Universitas Gadjah Mada Fakultas Kedokteran

. Marcellus

Universitas Gadjah Mada Fakultas Kedokteran

.Gunadi ( $\nabla$ drgunadi@ugm.ac.id)

Universitas Gadjah Mada Fakultas Kedokteran https://orcid.org/0000-0002-4707-6526

\section{Case report}

Keywords: COVID-19, emergency high ligation, incarcerated inguinal hernia, pediatric case, SARS-Cov-2, tertiary protection regulations

Posted Date: August 11th, 2020

DOl: https://doi.org/10.21203/rs.3.rs-40812/v1

License: (c) (i) This work is licensed under a Creative Commons Attribution 4.0 International License.

Read Full License 


\section{Abstract \\ Background}

SARS-Cov-2 infects not only adults, but also children, including pediatric surgery patients with acute abdomen. Here, we report a pediatric surgery case with incarcerated inguinal hernia and suspected COVID-19.

\section{Case presentation:}

A 11-month-old male was brought to our emergency department with the main complaint of recurrent yellowish-green vomiting that was experienced from one day before admission. High fever and shortness of breath were also reported. This patient was also suffering from moderate dehydration. Neither history of contact with a confirmed case of COVID-19 nor traveling from any local transmission area were found. However, a SARS-CoV-2 rapid antibody test revealed a positive result. A lump in the left scrotum that persisted during admission was found. Fluid resuscitation and nasogastric tube placement for decompression was performed. Manual reduction was attempted but failed to reduce the lump. Accordingly, we decided to perform an emergency high ligation using tertiary protection regulations, i.e., full personal protective equipment (PPE) for COVID-19. Intraoperatively, we found a small intestine loop trapped in the scrotum and stuck in the inguinal canal. Postoperatively, the baby was continued to be managed as a patient with COVID-19 while waiting for the real-time reverse transcription polymerase chain reaction (RT-PCR) results.

\section{Conclusions}

During the COVID-19 pandemic, surgeons should always be aware of the possibility of crosstransmission from the patient, since children are also susceptible to SARS-CoV-2 infection. When and wherever possible, surgeons should perform the procedure in the quickest and most effective manner to shorten exposure time with patient and anesthetic aerosols as well as using appropriate PPE.

\section{Background}

The ongoing outbreak of coronavirus disease 2019 (COVID-19), which is caused by severe acute respiratory syndrome coronavirus 2 (SARS-CoV-2), has spread throughout the world. Until June 17, this global pandemic has infected more than 8 million people in over 213 countries/territories and caused 440,290 deaths [1]. Rapid transmission of the virus can make hospitals overwhelmed, causing non-urgent operations to be delayed or even canceled [2]. This change of surgery scheduling is important in order to increase hospital bed capacity and maintain the availability of sufficient personal protective equipment (PPE) for health workers [3]. However, at the same time, pediatric surgery services have become severely affected [4]. For patients who experience life-threatening conditions, although surgical procedures in 
children with SARS-CoV-2 infection can pose risks of cross-transmission, the choice of an emergency operation may be the only lifesaving method when conservative treatment fails [5]. Here, we report a pediatric surgery case with incarcerated inguinal hernia and suspected COVID-19.

\section{Case Presentation}

A 11-month-old male was brought to our emergency department with the main complaint of recurrent yellowish-green vomiting that was experienced from one day before admission. The patient's abdomen was bloated, while the last defecation was the day before coming to the hospital. High fever and shortness of breath were also reported. On physical examination, his general condition was lethargic, and body weight was $7 \mathrm{~kg}$. Vital signs revealed pulse of 140 times per minute, respiratory rate 40 times per minute, body temperature of $39.5^{\circ} \mathrm{C}$, and capillary refill time $<2$ seconds. In the head examination, a sunken fontanel and eyes were found, indicating moderate dehydration. His abdominal wall was distended, and auscultation revealed an increase in bowel sounds, without any abdominal tenderness. A lump in the left scrotum that persisted during admission was found (Fig. 1). The lump had come and gone for 10 days. Routine laboratory findings showed leukocytosis, while the babygram revealed dilated bowel loops which suggested ileus (Fig. 2). Neither history of contact with a confirmed case of COVID-19 nor traveling from any local transmission area were found. However, a SARS-CoV-2 rapid antibody test revealed a positive result. Fluid resuscitation and nasogastric tube placement for decompression was performed. Manual reduction was attempted but failed to reduce the lump. Accordingly, we decided to perform an emergency high ligation using tertiary protection regulations, i.e., with full PPE for COVID-19. Intraoperatively, we found a small intestine loop trapped in the scrotum and stuck in the inguinal canal. Neither necrosis nor perforation were found in the intestinal loop. The intestine was returned into the peritoneal cavity followed by a high ligation. Postoperatively, the baby was continued to be managed as a patient with COVID-19 while waiting for the real-time reverse transcription polymerase chain reaction (RTPCR) results.

\section{Discussion}

Manual reduction is standard treatment for incarcerated inguinal hernia in children. Moreover, this technique is very useful to decrease the need for any emergency procedure during the in COVID-19 pandemic [6]. However, manual reduction is not without some risks. Several complications may occur following manual reduction, including peritonitis and bowel rupture [7]. The successful rate of manual reduction is about $70 \%$, therefore, if the manual reduction fails, an emergency surgery is mandatory [7].

During the COVID-19 pandemic, all medical procedures require clarity of the patient's status including whether infected with COVID-19. Along with proper precautions, great care must be taken during surgery to minimize the risk of cross infection to health workers. This includes PPE and control of aerosols or body fluids produced during operations that might be contaminated [8]. Besides the thoracic X-rays, preoperative COVID-19 screening using rapid antibody tests has become a protocol in the surgery department of our hospital, particularly for emergency patients. Our patient showed a positive result of 
the rapid antibody test for COVID-19. In our institution, the RT-PCR results will be obtained four days after the swab samples are taken. However, in this case, the surgical time window was limited and the patient deteriorated after failure of manual reduction. Accordingly, we decided to perform an emergency high ligation using tertiary protection regulations, i.e., with full PPE for COVID-19, while waiting for the RT-PCR results. The final RT-PCR results of the patient were negative for two consecutive swab tests. In spite of this, there is a possibility of false negative results of RT-PCR in patients with COVID-19 [9].

High fever in our patient could be caused by ileus complications due to fluid sequestration to the third cavity which results in dehydration and may be accompanied by bacterial translocation [10]. It might be also caused by the body's reaction to the SARS-CoV-2 infection [11-13]. In addition, shortness of breath in our patient might be caused by abdominal distension due to bowel dilatation [14] or by SARS-CoV-2 infection of the lungs [15].

The use of operating rooms specifically for patients with COVID-19 which are separated from non-COVID19 patients is also one way to reduce the risk of nosocomial infections [16]. In our hospital, we have prepared a containment area specifically designed as an emergency operating room according to strict patient and personnel safety standards. We use separate pathways for the patient and health workers to enter the operating theater, and our operating room is also equipped with negative pressure. These measures are in accordance with recommendations from Liu et al. [17].

The surgical procedures for open high ligation in children before and during the COVID-19 pandemic are the same. However, considering the possibility of the surgeons' and other surgical team members' exposure time to anesthetic aerosols and body fluids that might be contaminated with the SARS-Cov-2, it is recommended to perform the operation in the quickest and most efficient manner [17]. Furthermore, post-operative follow-up should strictly obey the tertiary protocol regulations by using full PPE [18].

\section{Conclusions}

During COVID-19 pandemic, surgeons should always be aware of the risk of cross-transmission from the patient, since children are also susceptible to SARS-CoV-2 infection. When and wherever possible surgeons should perform the procedure in the quickest and most effective manner to shorten exposure time with the patient and anesthetic aerosols as well as using appropriate PPE.

\section{List Of Abbreviations}

COVID-19: Coronavirus disease 2019, SARS-CoV-2: Severe acute respiratory syndrome coronavirus 2; PPE: Personal protective equipment

\section{Declarations}

\section{Ethics approval and consent to participate}


The Ethical Committee of the Faculty of Medicine, Public Health and Nursing, Universitas Gadjah Mada/Dr. Sardjito Hospital ruled the study exempt from approval because this study was a case report. The authors attest that full and informed consent was obtained from the patients who had undergone medical treatment in our hospital.

\section{Consent for publication}

Written and informed consent was obtained from the parents of the patient for publication of this case report and the associated images.

\section{Availability of data and material}

The datasets used and/or analyzed during the current study are available from the corresponding author on reasonable request

\section{Competing interests}

The authors declare that they have no competing interest.

\section{Funding}

The authors declare that they have no funding source.

\section{Authors' contributions}

$\mathrm{MM}, \mathrm{AP}$, and $\mathrm{G}$ conceived the study. $\mathrm{MM}$ and $\mathrm{ARF}$ drafted the manuscript, $\mathrm{KA}, \mathrm{M}$, and $\mathrm{G}$ critically revised the manuscript for important intellectual content. MM, AP and $\mathrm{G}$ facilitated all project-related tasks. All authors have read and approved the manuscript and agreed to be accountable for all aspects of the work in ensuring that questions related to the accuracy or integrity of any part of the work are appropriately investigated and resolved.

\section{Acknowledgments}


We are thankful to all staff and nurses who have taken care of the patient. This case report was funded by the Indonesian Ministry of Research and Technology/National Agency for Research and Innovation.

\section{References}

1. World Health Organization. Coronavirus Disease 2019 (COVID-19) situation report - 149. https://www.who.int/emergencies/diseases/novel-coronavirus-2019/situation-reports Accessed on June 17, 2020.

2. De Simone B, Chouillard E, Di Saverio S, Pagani L, Sartelli M, Biffl WL, et al. Emergency surgery during the COVID-19 pandemic: what you need to know for practice. Ann Roy Coll Surg. 2020;102:323-32.

3. Myles PS, Maswime S. Mitigating the risks of surgery during the COVID-19 pandemic. Lancet. 2020.

4. Polites SF, Azarow KS. Perspectives on pediatric appendicitis and appendectomy during the SARSCoV-2 pandemic. J Laparoendosc Adv Surg Tech A. 2020;30:356-7.

5. Zhou Y, Xu H, Li L, Ren X. Management for patients with pediatric surgical disease during the COVID19 epidemic. Ped Surg Int. 2020:1-2.

6. East B, Pawlak M, de Beaux AC. A manual reduction of hernia under analgesia/sedation (Taxis) in the acute inguinal hernia: a useful technique in COVID-19 times to reduce the need for emergency surgery-a literature review. Hernia. 2020:1.

7. Yang XF, Yang XF, Liu JL. Acute incarcerated external abdominal hernia. Ann Transl Med. 2014;2:110.

8. Stabilini C, East B, Fortelny R, Gillion JF, Lorenz R, Montgomery A, et al. European Hernia Society (EHS) guidance for the management of adult patients with a hernia during the COVID-19 pandemic. Hernia. 2020:1.

9. Xiao AT, Tong YX, Zhang S. False-negative of RT-PCR and prolonged nucleic acid conversion in COVID-19: rather than recurrence. J Med Virol. 2020.

10. Levinson H, Rimon A, Scolnik D, Amarilyio G, Glatstein M. Fever as a presenting symptom in children evaluated for ileocolic intussusception: the experience of a large tertiary care pediatric hospital. Ped Emerg Care. 2019;35:121-4.

11. Lu X, Zhang L, Du H, Zhang J, Li YY, Qu J, et al. SARS-CoV-2 infection in children. N Engl J Med. 2020;382:1663-5.

12. Wu Q, Xing Y, Shi L, Li W, Gao Y, Pan S, et al. Co-infection and other clinical characteristics of COVID19 in children. Pediatrics. 2020;e20200961.

13. Parri N, Lenge M, Buonsenso D. Children with COVID-19 in pediatric emergency departments in Italy. N Engl J Med. 2020;NEJMc2007617.

14. Hijaz NM, Friesen CA. Managing acute abdominal pain in pediatric patients: current perspectives. Pediatric Health Medicine Therapeutics. 2017;8:83.

15. Song W, Li J, Zou N, Guan W, Pan J, Xu W. Clinical features of pediatric patients with coronavirus disease (COVID-19). J Clin Virol. 2020;104377. 
16. Tan Z, Phoon PH, Jing F, Ting LX. Response and operating room preparation for the COVID-19 outbreak: a perspective from the National Heart Centre Singapore. J Cardiothorac Vasc Anesth. 2020;S1053-0770(20):30300-1.

17. Liu Z, Zhang Y, Wang X, Zhang D, Diao D, Chandramohan K, Booth CM. Recommendations for surgery during the novel coronavirus (COVID-19) epidemic. Indian J Surg. 2020;1-5.

18. Poulose BK, Phieffer LS, Mayerson J, Like D, Forrest LA, Rahmanian A, et al. Responsible return to essential and non-essential surgery during the COVID-19 pandemic. Gastrointest Surg. 2020;1-3.

\section{Figures}

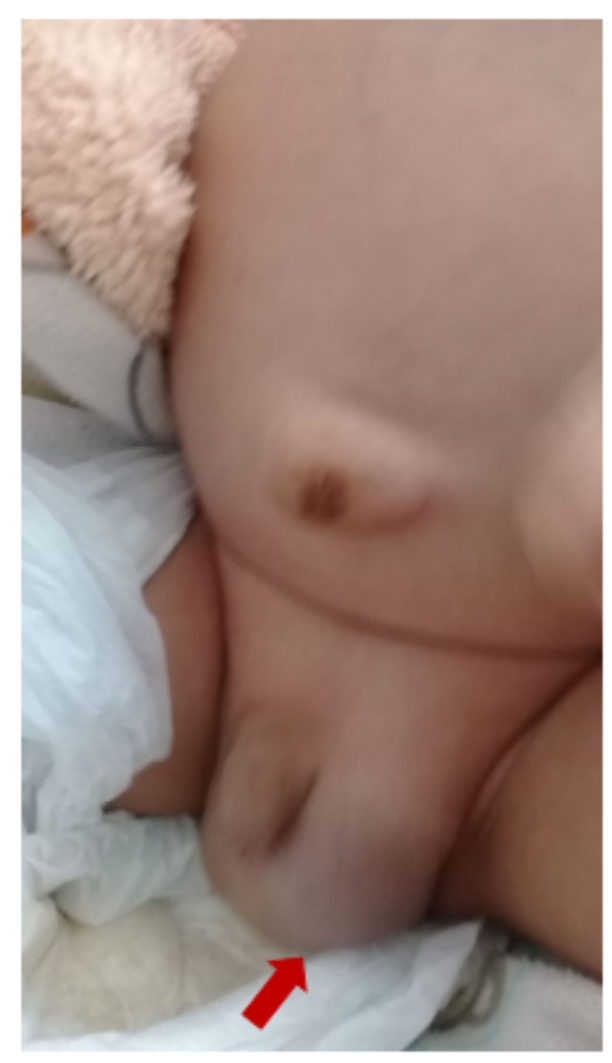

\section{Figure 1}

A lump was noted in the left scrotum. 


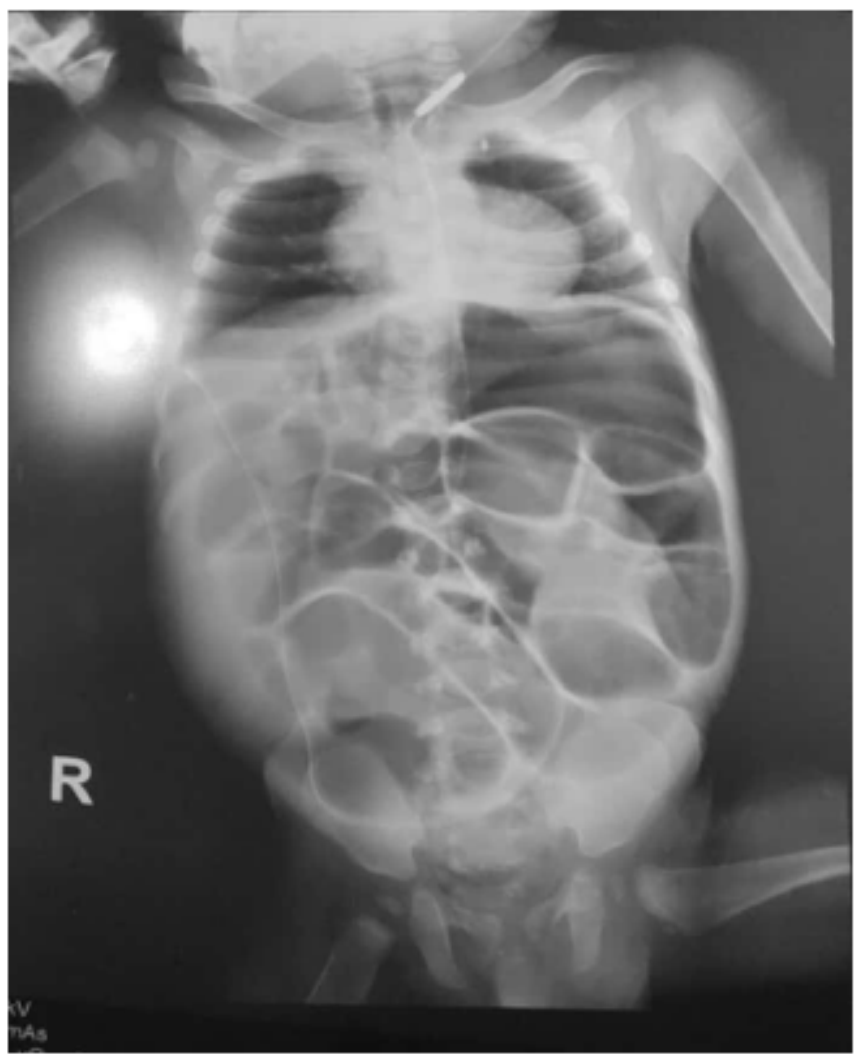

Figure 2

Babygram showed dilated bowel loops, suggesting ileus.

\section{Supplementary Files}

This is a list of supplementary files associated with this preprint. Click to download.

- CAREchecklistEnglishMunawir.pdf 